\title{
Estudio retrospectivo de las complicaciones de los catéteres temporales para hemodiálisis
}

\author{
Rodolfo Crespo Montero - María Dolores Contreras Abad - Rafael Casas Cuesta - Inmaculada Muñoz \\ Benítez - María Carmen Moreno Delgado - Lourdes Suanes Cabello
}

Enfermeros

Servicio de Nefrología del Hospital Reina Sofía de Córdoba.

\section{Resumen}

Los objetivos de este estudio fueron analizar la duración y las causas de retirada de los catéteres temporales para hemodiálisis

Hemos estudiado 1409 catéteres que fueron implantados en 608 pacientes en hemodiálisis periódicas $(60.9 \pm 15.5$ años, 341 mujeres (56\%) y 267 hombres (44\%). 280 (46\%) pacientes recibieron 1 catéter y $144(24 \%) 2$ catéteres. Posteriormente se analizaron 546 catéteres retirados exclusivamente por complicaciones.

La duración media de todos los catéteres $(n=546)$ fue de $21 \pm 36$ días. Las causas más frecuentes de retirada de catéteres por complicaciones fueron: $75 \%$ por déficit de flujo sanguíneo, $11 \%$ por infección, $6 \%$ por pérdida de sutura, $3 \%$ por oclusión, $3 \%$ por acodamiento. El déficit de flujo apareció a los $18 \pm 34$ días y la infección $29 \pm 50$ días. La duración media de los catéteres implantados en el lado izquierdo fue $16 \pm 32$ días y para el lado derecho $23 \pm 38$ días $(p<0.0001)$.

Cuando analizamos la duración de los catéteres entre las distintas venas utilizadas, la duración media fue

Correspondencia:
Rodolfo Crespo Montero
Email: rodo.crespo@gmail.com
Servicio de Nefrología del Hospital Reina Sofía de Córdoba
Avda. Menéndez Pidal s/n
14005 - CóRDOBA

de $25.3 \pm 43$ días para la yugular, $17.5 \pm 23$ para la subclavia, y $11.9 \pm 10$ días para la femoral, siendo las diferencias estadísticamente entre la yugularsubclavia y la vena femoral $(p<0.05)$. Cuando comparamos la duración entre los de una sola luz frente a los de doble luz, en los catéteres retirados por complicaciones no se encuentran diferencias significativas (análisis multivariante).

Podemos concluir, que la duración de los catéteres temporales colocados en vena yugular o subclavia y en el lado derecho, es significativamente superior a los colocados en vena femoral o lado izquierdo. No encontramos diferencias en la duración entre los catéteres de una o doble luz, al menos en nuestro estudio.

\section{PALABRAS CLAVE: \\ - HEMODIÁLISIS \\ - ACCESOS VASCULARES \\ - CATÉTERES TEMPORALES \\ - COMPLICACIONES}

\section{Retrospective study of the complications of temporary catheters for haemodialysis}

\section{Abstract}

The aim of this study was to analyze the duration and causes of removing complications for temporary catheters for haemodialysis. 
We studied 1409 catheters which were implanted on 608 patients in haemodialysis chronic $(60.9 \pm 15.5$ years, 341 females (56\%) and 267 males (44\%). $280(46 \%)$ patients received 1 catheter and 144 (24\%) 2 catheters. We analyzed 546 catheters retired exclusively by complications.

Mean duration of the catheters $(n=546)$ of was $21 \pm 36$ days. The main catheters complications that forced their retreat were: $75 \%$ for deficit blood flow rate, $11 \%$ infection, $6 \%$, for loss of surgical suture, $3 \%$ for occlusion, $3 \%$ for elbowed. The deficit blood flow rate appeared to the $18 \pm 34$ days and the la infection to the $29 \pm 50$ days. Mean duration of the catheters was of the $16 \pm 32$ days for the left side and $23 \pm 38$ for right side $(p<0.0001)$. When we compared the different blood vessel, mean duration of the catheters was of the $25.3 \pm 43$ days for the jugular, $17.5 \pm 23$ for the subclavia, and $11.9 \pm 10$ days for the femoral, being significant differences among jugular-subclavia and femoral vein $(p<0.05)$. When comparing the duration, among a single lumen vs dual lumen, in catheters retired by complications, they were not significant differences (multivariate analysis).

We can conclude that the duration, of the catheters placed in jugular or subclavia veins and right side is superior to the placed in femoral vein or left side, being recommended its use therefore as temporary access in HD, also facilitating the patient's mobility and ambulatory dialysis. We have not found, at least in our study, differs in the duration among the single lumen versus dual lumen catheters.

\section{KEY WORDS:}

- HAEMODIALYSIS

- VASCULAR ACCESS

- TEMPORARY CATHETERS

- COMPLICATIONS mente en la última década, debido al aumento de la edad y comorbilidad de la de los pacientes, y a la inclusión en programa de HD de cada vez más pacientes, sin acceso vascular previo ${ }^{1}$. Aunque se está generalizando el uso de CVC tunelizados, incluso para periodos de duración intermedios, los CVC temporales (CVCt) son utilizados con relativa frecuencia en pacientes con fracaso renal agudo y en pacientes crónicos que precisan HD urgente, o con fracaso temporal del acceso vascular permanente ${ }^{2}$. Sin embargo, estos catéteres no están exentos de complicaciones y su duración es limitada, señalándose como principales complicaciones, infección, déficit de flujo sanguíneo, coagulaciones y trombosis ${ }^{3,4}$. La infección es la complicación más grave, por cuanto puede ocasionar bacteriemia y sepsis en los pacientes, originando un cuadro severo, siendo su frecuencia del 4 al $20 \%$, dependiendo de las series publicadas ${ }^{5,6}$. El déficit de flujo sanguíneo es una complicación frecuente, que puede comprometer la eficacia de la HD y sobre todo, supone uno de los problemas más importantes para enfermería en su manejo. De hecho, se ha señalado el déficit de flujo de sangre como la primera causa de retirada de $\mathrm{CVCt}^{7}$.

Un factor que podría influir en la duración de los CVCt, es el tipo de catéter, es decir, el que sea de una sola o de doble luz, aunque no hemos encontrado estudios que analicen este factor en relación a la duración del mismo. En nuestro centro, se han venido utilizando indistintamente, CVCt de una luz y de doble luz.

Sin embargo y a pesar que su utilización se ha incrementado en los últimos años, no existen muchos estudios que analicen la duración de los CVCt y la causa de las complicaciones.

Por todo ello, nos planteamos los siguientes objetivos:

1. Examinar cuales habían sido las causas de retirada de todos los CVCt para HD implantados en nuestra unidad.

2. Analizar la duración y causas de retirada de todos los CVCt por complicaciones.

El uso de catéteres venosos centrales (CVC) para hemodiálisis (HD) se ha incrementado notable- 


\section{Material y métodos}

\section{Pacientes y recogida de datos}

Hemos realizado un estudio observacional retrospectivo de todos los CVCt implantados en nuestro Servicio, entre 1997 y 2009, a pacientes crónicos que iniciaban HD sin acceso vascular permanente o por fracaso temporal de este.

Todos los datos se han ido recogiendo en un registro electrónico que se diseñó al efecto, puesto que en nuestra unidad se implantan y recambian todos los CVCt que precisan los pacientes de la provincia, al ser centro de referencia de seis centros periféricos de diálisis. Con este registro se ha realizado un seguimiento de todos los catéteres aunque los pacientes no se dializaran en nuestra unidad. Se registraba la fecha de su colocación y se realizaba el seguimiento de los mismos hasta la fecha de retirada, recogiendo los datos del paciente, el tipo de catéter, vena de implantación, días de duración, complicaciones durante la implantación y causas de retirada.

El tipo de catéter y vena de implantación fueron decididos por el nefrólogo responsable de la colocación de los mismos y según necesidades de cada paciente.

Los motivos de retirada recogidos en el registro fueron: acodamiento o rotura del catéter, déficit de flujo sanguíneo, oclusión de alguna de las ramas o vías, fin de tratamiento, por pasar a diálisis peritoneal, por tener un acceso vascular definitivo, por pérdida de la sutura de fijación a la piel, por infección y por otras causas sin especificar (éxitus, cambio de vena de implantación sin que fuera por complicación, salida accidental del catéter).

\section{Catéteres y protocolo de cuidados}

Los CVCt utilizados fueron de poliuretano termosensible, de doble luz de 11 French de grosor y de 15,20 cm. de longitud; y de una sola luz de 8 French de grosor, de 15 y $20 \mathrm{~cm}$. de longitud (VasCath, Bard ${ }^{\circledR}$, EEUU). Para la vena femoral se utilizaron de $20 \mathrm{~cm}$.de longitud. Para la vena yugular derecha se utilizaron de $15 \mathrm{~cm}$ y para la yugular izquierda de $20 \mathrm{~cm}$. Se utilizaron indistintamente los catéteres de una o dos vías, según criterio del nefrólogo que lo colocaba o recambiaba.

Todos los CVCt fueron colocados o recambiados bajo las mismas condiciones de asepsia. En todas las unidades de diálisis dependientes de nuestro Servicio se siguió el mismo protocolo de conexión, utilización y desconexión de catéteres temporales, haciendo especial hincapié en las medidas de asepsia y el uso te guantes estériles y mascarillas en el personal de enfermería (Anexo I). El sellado del catéter se realizó con heparina sódica al $1 \%$ sin diluir, en una cantidad equivalente al volumen de cebado del mismo. Los catéteres solo fueron utilizados para hemodiálisis.

\section{Anexo 1. \\ UTILIZACIÓN DE LOS CATÉTERES TEMPORALES PARA HEMODIÁLISIS}

\section{OBJETIVO}

Utilizar catéter venoso de poliuretano TEMPORAL como acceso vascular para dializar al paciente, con las máximas medidas de asepsia.

\section{MATERIAL}

- Guantes

- Mascarillas

- Gasas

- Paños estériles

- Pinzas estériles

- Jeringa con solución salina heparinizada

- Heparina al $1 \%$

- Solución antiséptica de povidona iodada al $10 \%$

- Jeringas de 2-5, 10-20 ml.

\section{PROCEDIMIENTO}

- Colocación de mascarilla en la conexión y desconexión del paciente.

- Retirar el apósito que cubre el catéter; observando si presenta algún síntoma de infección (enrojecimiento, exudado purulento, calor, dolor). En caso de sospecha de infección, tomar muestra del exudado para cultivo y comunicar al médico.

- Colocación de guantes estériles.

- Retirar tapones y mantener pinzadas las conexiones externas mientras no esté conectada la jeringa para aspiración.

- Aspirar con una jeringa pequeña (2-5 ml) en ambas vías, arterial y venosa, comprobando su permeabilidad. En los catéteres de unipunción se aspirará una de las vías. La sangre extraída se desechará.

- Comprobar la permeabilidad con una nueva jeringa con suero salino heparinizado, introduciendo enérgicamente unos $10 \mathrm{ml}$, por cada una de la vías en catéteres de bipunción, y $10 \mathrm{ml}$, en la vía venosa de los catéteres de unipunción.

- Conectar la línea arterial del circuito sanguíneo a la vía arterial del catéter y poner la bomba de sangre en marcha, hasta completar el cebado del circuito con sangre.

- Conectar el extremo distal de la línea de sangre venosa a la vía venosa del catéter e iniciar la sesión de forma habitual.

- Ajustar el flujo sanguíneo al máximo que permita el catéter.

- Fijar las líneas de sangre con esparadrapo para evitar tracciones.

- Limpiar la zona de implantación y la parte externa del catéter con suero salino al $20 \%$ y secar, teniendo en cuenta el estado del orificio. 
Anexo 1. (cont.)

- Desinfectar la misma zona con una solución de povidona iodada.

- Colocar un apósito pequeño $(6 \times 7 \mathrm{~cm}$.), cubriendo la zona de inserción del catéter.

- Finalizada la sesión, retornar la sangre de forma habitual.

- Desconectar las líneas de sangre del catéter.

- Limpiar ambas vías del catéter con una embolada de suero fisiológico heparinizado

- Anticoagular con heparina al $1 \%$ en cantidad igual al volumen de cebado que indica el fabricante en cada conexión externa.

- Pinzar las conexiones externas de ambas vías con sus pinzas.

- Cerrar los orificios externos con sus respectivos tapones.

- Cubrir todo el catéter con apósitos y gasa adhesiva, fijándolo de la manera más cómoda para el paciente.

\section{OBSERVACIONES}

- El volumen de cebado en los catéteres de BIPUNCIÓN es de 0.9 $\mathrm{ml}$ en la vía arterial y $1.0 \mathrm{ml}$ en la vía venosa, para los catéteres de $15 \mathrm{~cm}$. Para los catéteres de $20 \mathrm{~cm}$. es de $1.1 \mathrm{ml}$ para la vía arterial y $1.2 \mathrm{ml}$ para la vía venosa.

- El volumen de cebado en los catéteres de UNIPUNCIÓN es de $1.75 \mathrm{ml}$ en la vía venosa, para los catéteres de $15 \mathrm{~cm}$. Para los catéteres de $20 \mathrm{~cm}$. es de $1.85 \mathrm{ml}$ para la vía venosa.

- En los catéteres de POLIURETANO está contraindicado utilizar soluciones alcohólicas como antiséptico.

- No realizar NUNCA una conexión a un catéter alto (jeringa, línea de sangre) sin estar cerrada la pinza de la extensión externa, porque puede producirse una embolia gaseosa.

La retirada del catéter por infección, fue decisión del nefrólogo responsable, siempre que el paciente presentó fiebre no atribuible a otras causas, infección local en el orificio de inserción o ambas situaciones a la vez.

Se definió el déficit de flujo como imposibilidad de conseguir un flujo sanguíneo de $200 \mathrm{ml} / \mathrm{min}$ en bipunción y $125 \mathrm{ml} / \mathrm{min}$ en unipunción, momento en el que el catéter era recambiado por otro nuevo.

\section{Análisis estadístico}

Para el análisis estadístico se ha realizado la distribución de frecuencias para las distintas variables cualitativas y la media \pm la desviación estándar de la media para las variables cuantitativas. Para la comparación de medias se utilizó la prueba t de Student para datos no pareados, y para la comparación de más de dos muestras se utilizó la prueba ANOVA con el método de Tukey para identificar los grupos diferentes. Las distintas correlaciones se realizaron con el test de Pearson. Se realizó análisis multivariante mediante regresión lineal múltiple. Se aceptó significación estadística para el valor de $p<0.05$. El análisis estadístico se ha realizado con un paquete estadístico SSPS 17.0.

\section{Resultados}

Se han revisado un total de 1460 CVCt: 950 de una luz y 510 de doble luz, de los cuales 1180 fueros de $15 \mathrm{~cm}$. y 280 de $20 \mathrm{~cm}$. de longitud. Fueron implantados 1061 en lado derecho y 374 en lado izquierdo. La distribución por venas utilizadas fue: 970 (67\%) en vena yugular ( $73 \%$ en lado derecho y $27 \%$ en el izquierdo), 25 en vena subclavia ( $2 \%$ ) y 449 en vena femoral $(31 \%)$.

Fueron implantados en 608 pacientes en HD, con una edad media de $61.2 \pm 15$ años, 279 mujeres (44\%) y 354 hombres (56\%). 280 (46\%) pacientes recibieron 1 catéter, 144 (24\%) pacientes recibieron 2 catéteres y $184(30 \%)$ recibieron más de 3 catéteres.

En la tabla I se exponen las causas de retirada de todos los CVCt analizados.

\begin{tabular}{|l|c|c|}
\hline CAUSAS DE RETIRADA & FRECUENCIA & PORCENTAJE \\
\hline Por tener acceso vascular permanente & 486 & $33 \%$ \\
\hline Déficit de flujo sanguíneo & 412 & $29 \%$ \\
\hline Otras causas & 301 & $21 \%$ \\
\hline Por fin de tratamiento & 105 & $7 \%$ \\
\hline Infección & 61 & $4 \%$ \\
\hline Trombosis de alguna rama o completa & 22 & $2 \%$ \\
\hline Pérdida de sutura & 39 & $3 \%$ \\
\hline Por pasar a diálisis peritoneal & 14 & $1 \%$ \\
\hline Acodamiento o rotura del catéter & 16 & $1 \%$ \\
\hline
\end{tabular}

Tabla 1. Causas de retirada del total de CVC temporales revisados ( $\mathrm{n}$ $=1460$ )

Después de analizadas las causas globales de retirada de todos los catéteres, se retiraron del análisis estadístico 906 catéteres que no presentaron complicaciones (maduración acceso vascular definitivo, cambio de técnica, fin de tratamiento) analizándose los 554 restantes, retirados por complicaciones.

En los catéteres retirados exclusivamente por complicaciones, la duración media fue de $21 \pm 36$ días (mediana 13 días). 
La distribución de las causas de retirada por complicaciones está reflejada en la tabla II.

\begin{tabular}{|l|c|c|}
\hline CAUSAS DE RETIRADA & FRECUENCIA & PORCENTAJE \\
\hline Déficit de flujo sanguíneo & 416 & $75 \%$ \\
\hline Infección & 61 & $11 \%$ \\
\hline Pérdida de sutura & 39 & $7 \%$ \\
\hline Trombosis de alguna rama o completa & 22 & $4 \%$ \\
\hline Acodamiento o rotura del catéter & 16 & $3 \%$ \\
\hline
\end{tabular}

Tabla 2. Causas de retirada de CVC por complicaciones $(n=554)$

Respecto al tiempo en el cual aparecen las complicaciones, el déficit de flujo apareció a los $17 \pm 20$ días desde la implantación, la infección a los $22 \pm 17$ días y la pérdida de sutura a los $25 \pm 20$ días.

La duración media según la vena de implantación fue de $25.3 \pm 43$ días, para la vena yugular, de $17.5 \pm 23$ días, para la vena subclavia, y de $11.9 \pm 10$ días, para la vena femoral. Al comparar la duración entre estos tres troncos venosos, se encontraron diferencias estadísticamente significativas entre venas yugular y femoral $(p<0.05)$, y entre las venas subclavia y femoral $(p<0.05)$, no encontrándose diferencias entre la duración de las venas yugular y subclavia.

Respecto al lado utilizado la duración de los CVCt implantados en el lado derecho $(n=395)$ fue de $21.1 \pm 26$ días, y para el lado izquierdo $(n=150)$ fue de $16 \pm 32$ días, no existiendo diferencias estadísticamente significativa entre ambos lados.

Cuando se analizó la duración en función de las luces de los catéteres, los de doble luz $(n=215)$ tuvieron una duración de $16.7 \pm 17$ días y los de una sola luz $(n=337)$ de $21 \pm 58$ días, siendo estas diferencias estadísticamente significativas $(p<0.05)$.

Al analizar la duración de los catéteres en función de la longitud de los mismos, los catéteres de $15 \mathrm{~cm}$. $(n=430)$ tuvieron una duración de $21.3 \pm 30$ días y los de $20 \mathrm{~cm}$. ( $n=124)$ tuvieron una duración de 14.2 \pm 14 días, siendo estas diferencias estadísticamente significativas $(p<0.01)$.

No se encontraron diferencias en la duración de los catéteres entre ambos sexos. No se encontró relación entre la duración de los catéteres y los años de los pacientes, ser diabético o niveles de hemoglobina.

En el análisis de regresión lineal simple la duración total en días como variable dependiente y la edad, género, vena utilizada, lado de implantación, luz y longitud del catéter, ser o no diabético y niveles de hemoglobina (se tomaron del último control rutinario del paciente antes de la retirada del catéter) como variables predictoras, solo el lado de implantación $(p<0.049)$ y la vena utilizada $(p<0.0001)$ predijeron de forma significativa los valores de la duración del catéter ( $R 2$ corregida=0,36).

\begin{tabular}{|l|l|c|l|l|}
\hline Constante & 36,22 & \multicolumn{2}{|c|}{ Intervalos de confianza de 95\% } & significación \\
\hline Lado implantación & $-5,27$ & $-10,485$ & $-0,060$ & 0,049 \\
\hline Vena utilizada & $-11,43$ & $-16,49$ & $-6,370$ & 0,000 \\
\hline
\end{tabular}

\section{Discusión}

Los CVCt constituyen un acceso temporal eficaz en HD, siendo utilizados con relativa frecuencia en pacientes con dificultad para un acceso permanente. Como se ha señalado, plantean una serie de complicaciones y una duración limitada ${ }^{8}$. Aunque su uso se está limitando a cortos periodos de tiempo, porque se está estandarizando la utilización de los CVC tunelizados cuando se prevé periodos superiores a 2-3 meses sin fistula nativa o protésica, los CVCt se siguen utilizando como recurso inmediato en pacientes crónicos, por fracaso de acceso vascular fijo, sobre todo en servicios de nefrología donde no es posible otra alternativa de acceso vascular en corto periodo de tiempo. Por otro lado, aunque las diferentes guías de práctica clínica aconsejan cambiar los CVCt a partir de un periodo de tiempo concreto, dependiendo de la vena en la que está implantado3 ${ }^{3}$ la realidad asistencial, dificultades de algunas unidades para obtener con rapidez un acceso vascular permanente y el perfil actual del paciente HD, conlleva el mantenimiento de los mismos mientras no presenten complicaciones y la necesidad del paciente se mantenga. Por ello, el manejo y cuidados de los mismos es un aspecto muy importante hoy día en las unidades de diálisis, en el trabajo de enfermería?.

En nuestros resultados, podemos destacar el bajo índice de infecciones encontrado (11\%) en los CVCt 
retirados exclusivamente por complicaciones, similar al encontrado por otros autores, los cuales han destacado el papel de los cuidados de enfermería en este bajo índice de infecciones ${ }^{10,11}$. De hecho en nuestro servicio tenemos un procedimiento bastante estricto en cuanto a las medidas de asepsia, del manejo de todos los CVC, haciendo especial hincapié en la utilización, durante la conexión y desconexión de los mismos, de mascarilla por parte de la enfermera/o y paciente.

La mayor causa de retirada de CVCt por complicaciones fue el déficit de flujo sanguíneo para poder realizar una HD adecuada, resultados que coinciden con lo encontrado por otros autores ${ }^{12,13}$. En cuanto a la duración en días, es de destacar que comparando la duración de los CVCt implantados en vías altas (yugular-subclavia) frente a la vena femoral, se obtiene una diferencia significativa, algo común en la mayoría de series publicadas ${ }^{13-15}$.

Al comparar la duración de los CVCt de una sola luz frente a los de doble luz, presentan mayor duración los de unipunción, en el análisis bivariado, sin embargo en el análisis multivariante este factor no tiene influencia en la duración de los catéteres. Este dato no puede ser comparado con otros resultados, pues no existe bibliografía al respecto. Hay que indicar que el uso de catéteres de una sola luz para HD para unipunción no es muy habitual y su utilización puede considerarse residual, actualmente. En un estudio multicéntrico europe $0^{9}$, encontraron una mayor tasa de infecciones en los catéteres de bipunción, en comparación a los de una sola luz, aunque hay que tener en cuenta que la serie no era muy amplia.

Aunque la duración de los catéteres implantados en troncos venosos del lado derecho es mayor que en los implantados en el lado izquierdo, las diferencias no son significativas. Sin embargo, la duración de los CVCt de $15 \mathrm{~cm}$ es significativamente mayor que las de los catéteres de $20 \mathrm{~cm}$. Esto es lógico, pues los CVCt de 20 $\mathrm{cm}$ son los utilizados en el abordaje de la vena yugular interna izquierda y en las venas femorales, factores que intervienen en una menor duración, como hemos comentado anteriormente.

En cuanto al tiempo en el que aparecen las complicaciones, la aparición de déficit de flujo aparece se produce a los 18 días de media y la infección a los
29 días, resultados también en consonancia por lo publicado por otros autores ${ }^{16}$.

En el análisis de regresión lineal múltiple, la duración de los catéteres, ajustadas todas las variables, solo se asocia con una menor duración el lado de implantación izquierdo (5,27 días de duración media menos que en lado derecho y la vena femoral (11,43 días de duración media menos que en vena yugular).

Como principales conclusiones, podemos decir que la duración de los catéteres altos en vena yugular interna es superior a los femorales, por presentar menos incidencia de complicaciones; y que los implantados en el lado derecho duran más que el lado izquierdo. La causa más frecuente de retirada del catéter por complicaciones es el déficit de flujo sanguíneo.

Recibido: Diciembre 2010

Revisado: Diciembre 2010

Modificado: Enero 2011

Aceptado: Enero 2011

\section{Bibliografía}

1. Gruss E, Portolés J, Tato A et al. Repercusiones clínicas y económicas del uso de catéteres tunelizados de hemodiálisis en un área sanitaria. Nefrología 2009; 29(2):123-129.

2. Mendelssohn DC, Ethier J, Elder SJ et al. Haemodialysis vascular access problems in Canada: results from the Dialysis Outcomes and Practice Patterns Study (DOPPS II). Nephrol Dial Transplant; 2006; 21:721-728.

3. Sociedad Española de Nefrología. Guía del acceso vascular en hemodiálisis. Disponible http://www. senefro.org/modules/subsection/files/guia_acceso_ vascular.pdf?check_idfile=984 (consultado el 1505-2010).

4. Kairaitis LK, Gottlieb T. Outcome and complications of temporary haemodialysis catheters. Nephrol Dial Transplant 14: 1710-1714; 1999. 
5. Rayner HC, Pisoni RL, Bommer J, Canaud B, Hecking $E$, Locatelli $F$, et al. Mortality and hospitalization in haemodialysis patients in five European countries: Results from the Dialysis Outcomes and Practice Patterns Study (DOPPS). Nephrol Dial Transplant 2004; 19(1):108-20.

6. Oliver M, Callery S, Thorpe K, Schwab S, Churchill D. Risk of bacteremia from temporary haemodialysis catheters by site of insertion and duration of use: $A$ prospective study. Kidney Int; 2000; 58:2543-45.

7. Marcel C, Marc G, Vervloet and Piet M. Compared to tunnelled cuffed haemodialysis catheters, temporary untunnelled catheters are associated with more complications already within 2 weeks of use. Nephrol Dial Transplant. 2004; 19:670-677.

8. Arenas MD, Malek T, López-Collado M, Gill MT, Moledous A, Morales A, Cotilla E, Álvarez-Ude F. Operación retirada de catéteres venosos tunelizados en una unidad de diálisis. ¿Es posible cambiar la tendencia en el uso creciente de éstos? Nefrología 2009; 29 (4): 318-326.

9. Comité de Investigación EDTNA/ERCA. Complicaciones del acceso vascular. EDTNA/ERCA Journal, 2003, XXIX:3-7.

10. Hryszko T, Brzosko S, Mazerska M, Malyszko J. Risk factors of nontunneled noncuffed hemodialysis catheters malfunction. Nephron Clin Pract; 96:c43c47; 2004.
11. Ponikvar R, Buturovic- Ponikvar J. Temporary hemodialysis catheters as a long-term vascular access in chronic hemodialysis patients. Ther Apher Dial; 9(3):250-253; 2005.

12. Weijmer MC, Vervloet MG, Wee PM. Compared to tunnelled cuffed haemodialysis catheters, temporary untunnelled catheters are associated with more complications already within 2 weeks of use. Nephrol Dial Transplant; 2004, 19, 3:670-677.

13. Brzosko S, Hryszko T, Malyszko J, Malyszko JS, Mazerska M, Mysliwiec M. Femoral locatización and higher ultrafiltración rate but not concentration of heparin used for canal locking of hemodialysis catheter are negative predictors for its malfunction. Am J Nephrol; 2008; 28(2): 298-303.

14. Naumovic RT, Jovanovic DB, Djucanovic LJ. Temporary vascular catheters for hemodialysis: a 3-years prospective study. IntJ artif Organs; 2004; 27(10):848-854.

15. Lafrance JP, Rahma E, Lelorier J, Iqbal S. Vascular access-related infections: definitions, incidence rates, and risk factors. Am J Kidney Dis; 52(5):982993, 2008.

16. de Freitas LW, Neto MM, Nascimento MM, Figueiredo JF. Bacterial colonization in hemodialysis temporary dual lumen catheters: a prospective study. Ren Fail. 2008; 30(1):31-5. 


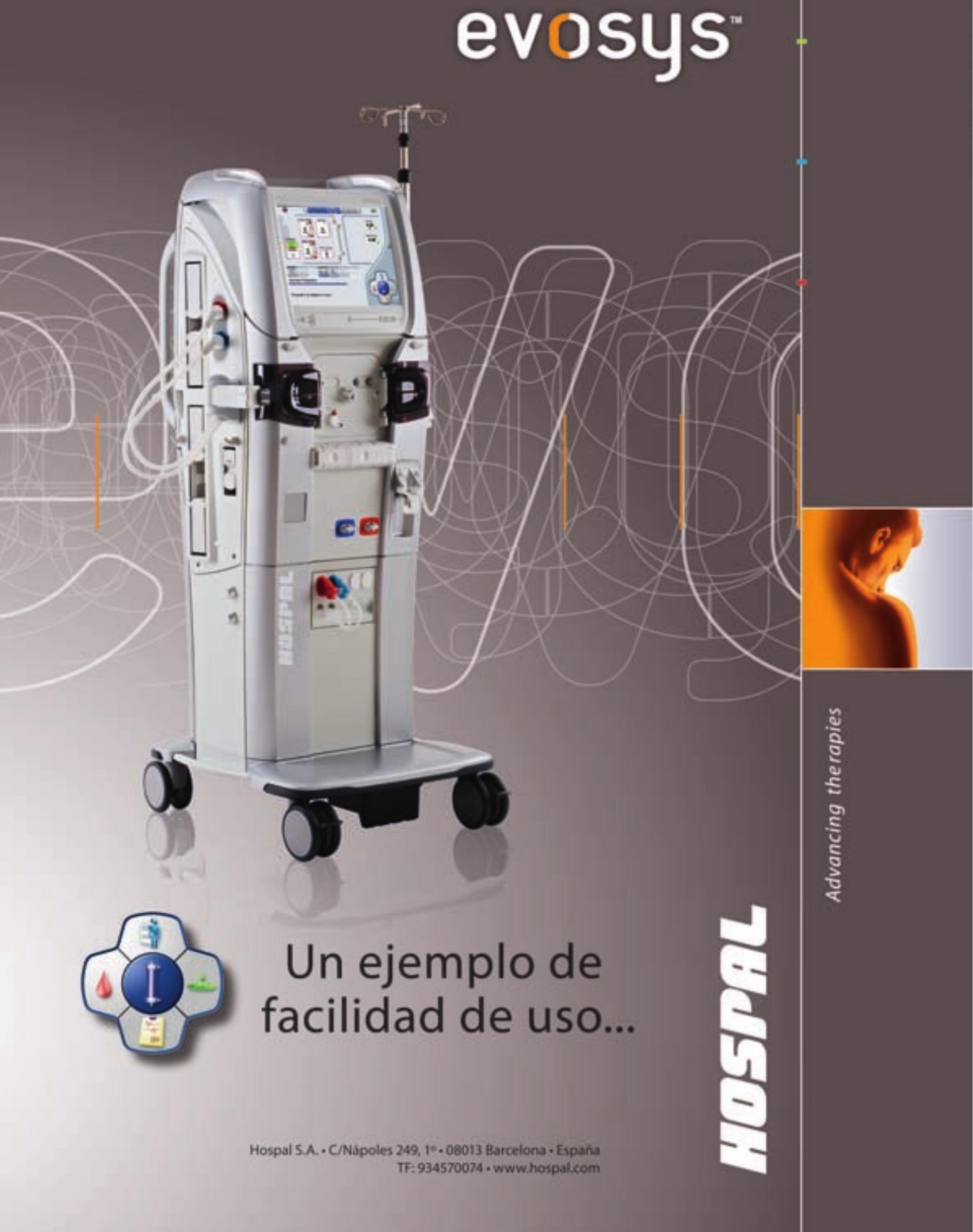

